\title{
Review of: "Role of anterior segment optical coherence tomography angiography in the assessment of acute chemical ocular injury: a pilot animal model study"
}

Jeniffer Jesus

Potential competing interests: The author(s) declared that no potential competing interests exist.

This is pilot animal prospective study developed in New Zealand white rabbits to investigate the role of anterior segment OCT angiography (AS-OCTA) in the assessment of limbal ischemia after acute chemical ocular trauma. The principal findings were the reduction in limbal vascularization $24 \mathrm{~h}$ after the chemical injury, and the positive correlation between the extent of limbal alkali exposure and the reduction of limbal vascular density. The main limitation of the study, which is well highlighted was the small sample size of the animal model.

The article is well written, with a good description of the methodology and clinical findings. The discussion of the results clearly shows the potential role of the analysis of conjunctival vascularization by AS-OCTA in the evolution of acute chemical trauma.

However, there are some concerns which include the following:

1. There is no characterization and description of the control group.

2. Chemical injury technique: "The conjunctival sac was then rinsed with saline until a $\mathrm{pH}$ of 7 to 7.5 was achieved. for 4 weeks to all eyes." - typo error. The conjunctival sac was rinsed for 4 weeks? After the chemical injury, the treatment included only antibiotics or also artificial tears and topical vitamin compounds?

3. There is no information regarding the healing period.

4. The follow-up period should be include observations at 3rd month and 6th month to observe what happens with this vascular increase over time.

5. It would be interesting to correlate the healing time with vascular density measurements. 